\title{
BMJ Open Comparison of coronary heart disease risk assessments among individuals with metabolic syndrome using three diagnostic definitions: a cross-sectional study from China
}

\author{
Juan Zhou, ${ }^{1,2}$ Qin Gao, ${ }^{1,2}$ Jun Wang, ${ }^{3}$ Min Zhang, ${ }^{4}$ Jianping Ma, ${ }^{4}$ Changyi Wang, ${ }^{4}$ \\ Hongen Chen, ${ }^{4}$ Xiaolin Peng, ${ }^{4}$ Liping Hao ${ }^{1,2}$
}

To cite: Zhou J, Gao Q, Wang J, et al. Comparison of coronary heart disease risk assessments among individuals with metabolic syndrome using three diagnostic definitions: a cross-sectional study from China. BMJ Open 2018;8:e022974. doi:10.1136/ bmjopen-2018-022974

\section{- Prepublication history for} this paper is available online. To view these files, please visit the journal online (http://dx.doi. org/10.1136/bmjopen-2018022974).

$\mathrm{XP}$ and LH contributed equally.

Received 17 March 2018

Revised 28 August 2018

Accepted 31 August 2018
Check for updates

(C) Author(s) (or their employer(s)) 2018. Re-use permitted under CC BY-NC. No commercial re-use. See rights and permissions. Published by BMJ.

For numbered affiliations see end of article.

Correspondence to Dr Xiaolin Peng; 25731738@qq.com and Dr Liping Hao;

haolp@mails.tjmu.edu.cn

\section{ABSTRACT}

Objective Metabolic syndrome (MetS) is a notable risk factor of coronary heart disease (CHD). However, there are differences in the methods used to define MetS. The purpose of this study was to determine which MetS definition most fully reflects the 10-year probability of CHD based on the Framingham risk algorithm.

Design Cross-sectional study.

Setting Data were obtained from the China Health and Nutrition Survey and the Influencing Factors of Chronic Diseases Survey conducted among residents of Nanshan District in Shenzhen, China.

Participants In total, 1721 participants aged $20-80$ years were included in this study.

Methods MetS was diagnosed according to the criteria of the National Cholesterol Education Program's Adult Treatment Panel (revised NCEP-ATP III), the International Diabetes Federation (IDF) and the Chinese Diabetes Society (CDS). The NCEP-ATP III algorithm was used to calculate the Framingham risk score, and the Framingham risk score was used to define the probability of developing CHD within 10 years either as low $(<6 \%)$, moderate $(6 \%-10 \%)$, moderately high $(10 \%-20 \%)$ or high $(>20 \%)$. Chi-square tests with or without the Bonferroni correction were used to compare the differences in the distribution of the 10-year estimated risk of developing CHD among the three definitions.

Results Compared with the other definitions, the revised NCEP-ATP III criteria identified more participants (30.96\%, $95 \% \mathrm{Cl} 28.8 \%$ to $33.2 \%$ ) as having MetS, while the CDS criteria showed the highest 10 -year probability of developing CHD. The 10-year probability of developing $\mathrm{CHD}$ in the participants with MetS was significantly higher than that in the participants without MetS (CDS: $\chi^{2}=157.65$, revised ATP III: $\chi^{2}=45.17$, IDF: $\chi^{2}=306.15$, all $\mathrm{p}<0.001$ ), and all definitions more fully reflect the CHD risk in men than in women (revised NCEP-ATP III: $\chi^{2}=72.83$; IDF: $\chi^{2}=63.60$; CDS: $\chi^{2}=23.84$; all $p<0.001$ ).

Conclusions This study demonstrates the differences in the prevalence and distribution of the 10-year estimated risk of developing CHD based on the definition of MetS. A significant finding of this study is that the MetS definitions have better performance for men than for women. Further studies in China, especially longitudinal studies, are
Strengths and limitations of this study

- The complex, multistage probability sample design is fairly representative of the Chinese population in Shenzhen.

- There was a low percentage of missing data in general.

- Three definitions of metabolic syndrome (MetS) were used to compare the discrepancy in the prevalence of MetS and the 10-year probability of developing coronary heart disease (CHD) based on the Framingham risk score.

- The Framingham algorithm may overestimate the risk of developing CHD in a Chinese population.

- This study adopted a cross-sectional design, and cohort studies are needed to further prove the predictive value and determine which MetS definition is the most predictive of the development of $\mathrm{CHD}$.

needed to determine which definition of MetS is best suited for predicting CHD risk.

\section{INTRODUCTION}

Metabolic syndrome (MetS) is typically diagnosed based on abnormalities in a specific set of clinical measures and is associated with an increased risk of developing coronary heart disease (CHD) ${ }^{1-3}$ A meta-analysis conducted by Mottillo et al showed that MetS is associated with an increased risk of cardiovascular outcomes and all-cause mortality. ${ }^{4}$ Another meta-analysis including 43 cohorts reported that the relative risk of cardiovascular events and deaths is 1.78 times greater in individuals with MetS. ${ }^{5}$ In addition, a matched cohort study found that participants with MetS have a 2.85-fold (2.27-3.57) and 1.80-fold (1.422.28) increase in CHD risk in the unadjusted and fully adjusted models, respectively. ${ }^{6}$ 
Currently, diverse methods are used to define MetS, including the 2002 US Third Report of the National Cholesterol Education Program (NCEP) Expert Panel on Detection, Evaluation, and Treatment of High Blood Cholesterol in Adults (Adult Treatment Panel (ATP) III), ${ }^{7}$ the 2005 International Diabetes Federation (IDF) criteria, ${ }^{8}$ the 2004 Chinese Diabetes Society (CDS) criteria $^{9}$ and the 2009 Joint Interim Statement (JIS) criteria. ${ }^{10}$ Although these criteria have similar components, there are also variations. For example, the criteria of the revised NCEP-ATP III and IDF are the same, but the IDF criteria include abdominal obesity as an obligatory component defining MetS. The CDS criteria consider the importance of each component equal and use the body mass index (BMI) rather than waist circumference (WC) as an index to define obesity. In addition, the cut-off values for specific components in the ATP III criteria differ from those in the revised NCEP-ATP III and IDF criteria, except for the cut-off value for triglyceride (TG) levels. Furthermore, the JIS criteria were created in a collaboration among global expert groups and are similar to the revised NCEP-ATP III criteria, including the national and regional cut-off values for WC.

The differences among these definitions of MetS have resulted in discrepancies in the reported prevalence of MetS among various populations and difficulties in identifying target populations for the prevention and control of MetS. Most importantly, since MetS is known to be a risk factor for developing CHD, knowledge regarding which MetS definition better reflects the risk of developing CHD is critical.

The present study aimed to investigate the discrepancy in the prevalence of MetS using three different definitions (the revised NCEP-ATP III, the IDF and the CDS criteria) in the Chinese population. This study also aimed to determine which MetS definition most fully reflects the 10-year probability of developing CHD based on the Framingham risk algorithm.

\section{METHODS}

\section{Subjects}

We combined data from parts of the China Health and Nutrition Survey to examine the association between health status and changes in economic and social conditions with data from the Influencing Factors of Chronic Diseases Survey. Briefly, this study comprised two cross-sectional studies conducted among residents of Nanshan District in Shenzhen, Guangdong Province in 2015. During the investigation, a complex, multistage probability sample design was used for the distribution of both surveys. In addition, the participants included in the survey were required to be eligible adults who had been living in Nanshan District for at least 6 months.

This study sample consisted of 1820 adults; however, 99 subjects were excluded because anthropometric or biochemical information needed for an accurate diagnosis of MetS was lacking. In total, 1721 participants aged
20 to 80 years old were ultimately eligible for analysis. All participants were informed of the specific details and provided informed consent before the surveys, both of which were approved.

\section{Patients and public involvement}

The patients were not directly involved in the design of the study nor in the recruitment and carrying out of the study. The results of this study will be disseminated to the study participants through different channels. First, we directly communicate with the Community Health Service Centre, which will provide the related results to the residents, especially patients with MetS. Second, the work will be published in an open-access peer-reviewed journal to provide everyone with the opportunity to obtain the information.

\section{Measurements}

A face-to-face interview was conducted by an investigator who was trained to administer both surveys. A standardised questionnaire was used to collect information regarding the participants' demographic characteristics, smoking status, drinking status, physical activity, medical history and medication use. Weight, height and WC were measured by an investigator using standard measurement methods. Weight and height were measured while the participants were marginally clothed without shoes using an SK-X80 (Sonka Corporation, Shenzhen, China) and recorded to the nearest $0.1 \mathrm{~kg}$. The BMI was calculated as weight in kilograms divided by the square of height in metres. The WC was measured to the nearest $0.1 \mathrm{~cm}$ at the midpoint between the lower rib and the iliac crest at the end of normal expiration while the participants were standing. Blood pressure was measured using a standard mercury sphygmomanometer with the cuff on the right upper arm after $5 \mathrm{~min}$ of rest. Three blood pressure readings were recorded, and the mean of the three readings was calculated.

\section{Laboratory tests}

The participants were required to fast overnight (at least 10 hours) before blood collection was conducted by the nurse. Blood was drawn from the vein in the morning at the Community Health Service Centre and transferred to the Shenzhen Nanshan Centre for Chronic Disease Control for further treatment within 2 hours of blood collection. The blood specimens were collected in a $5 \mathrm{~mL}$ EDTA vacuum tube for routine examination and $5 \mathrm{~mL}$ coagulation tubes for the biochemical analysis and stored in a cooler during transportation. Once the specimens arrived at the Department of Laboratory Medicine, they were centrifuged at $3000 \times \mathrm{g}$ for $10 \mathrm{~min}$ at room temperature instantaneously. The fasting blood glucose (FBG) level, total cholesterol (TC) level, TG level, low-density lipoprotein concentration (LDL-C) and high-density lipoprotein concentration (HDL-C) were analysed by an automatic clinical chemistry analyser (HITACH 7080, Tokyo, Japan). FBG, TC, TG, HDL-C and LDL-C were determined by enzymatic methods. 
Table 1 Definitions of metabolic syndrome (MetS)

\begin{tabular}{|c|c|c|c|}
\hline MetS components & $\begin{array}{l}\text { Revised NCEP-ATP III criteria } \\
\text { (three or more) }\end{array}$ & $\begin{array}{l}\text { IDF criteria } \\
\text { (central obesity and two or more) }\end{array}$ & $\begin{array}{l}\text { CDS criteria } \\
\text { (BMI and two or more) }\end{array}$ \\
\hline WC (BMI) & $W C \geq 90 / 80 \mathrm{~cm}(\mathrm{M} / \mathrm{W})$ & $W C \geq 90 / 80 \mathrm{~cm}(M / W)$ & $\mathrm{BMI} \geq 25 \mathrm{~kg} / \mathrm{m}^{2}$ \\
\hline SBP/DBP & $\geq 130 / 85 \mathrm{~mm} \mathrm{Hg}$ or MP & $\geq 130 / 85 \mathrm{~mm} \mathrm{Hg}$ or MP & $\geq 140 / 90 \mathrm{~mm} \mathrm{Hg}$ or MP \\
\hline FBG (mmol/L) & $\geq 5.6 \mathrm{mmol} / \mathrm{L}$ or MT & $\geq 5.6 \mathrm{mmol} / \mathrm{L}$ or $\mathrm{MT}$ & $\geq 6.1 \mathrm{mmol} / \mathrm{L}$ or $\mathrm{MT}$ \\
\hline TG (mmol/L) & $\geq 1.70 \mathrm{mmol} / \mathrm{L}$ & $\geq 1.70 \mathrm{mmol} / \mathrm{L}$ & \\
\hline HDL-C (mmol/L) & $<1.0 / 1.3 \mathrm{mmol} / \mathrm{L}(\mathrm{M} / \mathrm{W})$ & $<1.0 / 1.3 \mathrm{mmol} / \mathrm{L}(\mathrm{M} / \mathrm{W})$ & \\
\hline $\begin{array}{l}\mathrm{TG}(\mathrm{mmol} / \mathrm{L}) \text { and } \\
\mathrm{HDL}-\mathrm{C}(\mathrm{mmol} / \mathrm{L})\end{array}$ & & & $\begin{array}{l}\mathrm{TG} \geq 1.70 \mathrm{mmol} / \mathrm{L} \text { or }(\text { and }) \\
\mathrm{HDL}-\mathrm{C}<0.9 / 1.0 \mathrm{mmol} / \mathrm{L}(\mathrm{M} / \mathrm{W})\end{array}$ \\
\hline
\end{tabular}

BMI, body mass index; CDS, Chinese Diabetes Society; DBP, diastolic blood pressure; FBG, fasting blood glucose; HDL-C, high-density lipoprotein cholesterol; IDF, International Diabetes Federation; LDL-C, low-density lipoprotein cholesterol; M, men; MP, medication for blood pressure; MT, medication for blood glucose; NCEP-ATP, National Cholesterol Education Program's Adult Treatment Panel; SBP, systolic blood pressure; TG, triglycerides; W, women; WC, waist circumference.

\section{Definition of MetS and the Framingham risk algorithm}

In this study, we used the following three different definitions of MetS: the revised NCEP-ATP III Criteria for Asians (revised by the American Heart Association and the National Heart, Lung, and Blood Institute (AHA/ NHLBI) in $2005^{11}$ and are the same criteria as those used by the JIS in 2009, ${ }^{10}$ IDF criteria for Asians ${ }^{8}$ and CDS criteria. ${ }^{9}$ The details of the three criteria are provided in table 1. The Framingham risk score was calculated by using the NCEP-ATP III algorithm, ${ }^{7}$ which uses the following variables: sex, age, TC, smoking status, HDL-C and systolic blood pressure (SBP) (treatment for hypertension and SBP value). The 10-year probability of developing CHD was calculated based on the risk score by gender. In addition, we defined the 10 -year probability of developing CHD as low $(<6 \%)$, moderate $(6 \%-10 \%)$, moderately high $(10 \%-20 \%)$ and high $(>20 \%) .{ }^{12}$ Participants with pre-existing diabetes or self-reported cardiovascular disease (CVD) (including heart attack, heart failure or stroke) were distributed to the high-risk group. Diabetes was defined as having a fasting glucose level of $7.0 \mathrm{mmol} / \mathrm{L}$ after a 12 -hour fast, use of oral hypoglycaemic agents or insulin or self-reported diagnosis of diabetes.

\section{Statistical analysis}

Continuous variables with normal and skewed distributions are expressed as the means (SD) and medians (IQR), respectively. Categorical variables are reported as percentages, and the differences were compared using $\chi^{2}$ test with or without Bonferroni correction. First, the prevalence of MetS was calculated based on the three definitions of MetS, and the differences were compared. Second, the distribution of the 10-year estimated risk of developing CHD according to each of the three definitions of MetS was compared to determine which definition is the best predictor of CHD development. A two-sided $p$ value less than 0.05 was considered statistically significant. All statistical analyses were performed using SPSS software (V.20.0; SPSS, Chicago, Illinois, USA).

\section{RESULTS}

\section{Prevalence of MetS}

In total, 1721 participants aged 20 to 80 years were included in this study. The general characteristics of the participants are presented in table 2. The prevalence of MetS based on the definitions by the revised NCEP-ATP III, IDF and CDS criteria is presented in table 3 . The age-adjusted and sex-adjusted prevalence of MetS among participants aged 20 to 80 years according to the revised ATP III, IDF and CDS criteria was $30.96 \%, 19.93 \%$ and $10.88 \%$, respectively. The age-standardised prevalence of MetS among men aged 20 to 80 years according to the revised NCEP-ATP III, IDF and CDS criteria was $30.21 \%, 10.85 \%$ and $13.12 \%$, respectively, and that for women aged 20 to 80 years was $31.74 \%, 29.24 \%$ and $8.58 \%$, respectively. The difference in the prevalence of MetS based on the three definitions was large in both sexes. In particular, the prevalence of MetS based on the revised ATP III criteria in the women was 3.7-fold greater than that based on the CDS criteria.

The age-adjusted and sex-adjusted prevalence of MetS increased with age in those younger than 30 years to those older than 60 years from $17.78 \%$ to $36.1 \%, 9.26 \%$ to $35.93 \%$ and $0.44 \%$ to $23.17 \%$ based on the revised ATP III, IDF and CDS criteria, respectively. The age-specific prevalence in the women was found to be higher than that in the men according to the IDF criteria (women: $29.24 \%$ (95\% CI $26.4 \%$ to $32.1 \%$ ); men: $10.85 \%$ (95\% CI $8.6 \%$ to $13.2 \%)$ ), but the results were opposite using the CDS criteria (women: $8.58 \% \quad(95 \%$ CI $7.1 \%$ to $10.6 \%)$; men: $13.12 \%$ (95\% CI: $10.6 \%$ to $15.6 \%)$ ). An analysis stratified by age according to the revised ATP III criteria showed that the prevalence of MetS in men aged $<40$ years was higher than that in women in the same age group, while the reverse was true for those aged $\geq 50$ years (table 3 , figure 1 ). 
Table 2 Characteristics of the participants

\begin{tabular}{|c|c|c|c|}
\hline & Total $(n=1721)$ & Men $(n=716,41.6 \%)$ & Women $(n=1005,58.4 \%)$ \\
\hline Age (years) & $44.41 \pm 12.43$ & $45.23 \pm 12.47$ & $43.83 \pm 12.38$ \\
\hline Body mass index (kg/m²) & $23.68 \pm 3.31$ & $24.64 \pm 3.16$ & $23.00 \pm 3.24$ \\
\hline Waist circumference (cm) & $82.08 \pm 9.84$ & $86.91 \pm 9.03$ & $78.63 \pm 8.90$ \\
\hline Fasting blood glucose (mmol/L) & $5.29 \pm 1.22$ & $5.43 \pm 1.50$ & $5.19 \pm 0.96$ \\
\hline Triglycerides (mmol/L) & $1.47 \pm 1.21$ & $1.80 \pm 1.41$ & $1.23 \pm 0.98$ \\
\hline Total cholesterol (mmol/L) & $4.28 \pm 0.96$ & $4.31 \pm 0.96$ & $4.26 \pm 0.96$ \\
\hline $\mathrm{HDL}-\mathrm{C}$ (mmol/L) & $1.31 \pm 0.35$ & $1.15 \pm 0.31$ & $1.42 \pm 0.34$ \\
\hline LDL-C (mmol/L) & $2.59 \pm 0.80$ & $2.70 \pm 0.82$ & $2.50 \pm 0.78$ \\
\hline $\mathrm{SBP}(\mathrm{mm} \mathrm{Hg})$ & $118.46 \pm 16.19$ & $122.69 \pm 14.77$ & $115.45 \pm 16.49$ \\
\hline DBP (mm Hg) & $75.99 \pm 10.31$ & $79.58 \pm 9.72$ & $73.42 \pm 9.93$ \\
\hline Hypertension (\%) & 13.9 & 16.9 & 11.8 \\
\hline Diabetes (\%) & 5.3 & 6.8 & 4.2 \\
\hline Dyslipidaemia (\%) & 10.2 & 14.5 & 7.2 \\
\hline Current smoker (\%) & 5.7 & 13.0 & 5.1 \\
\hline Central obesity (\%) & 24.2 & 13.3 & 31.9 \\
\hline 10-year probability of developing CHD (\%) & $1(1,2)$ & $2(0,8)$ & $1(1,1)$ \\
\hline
\end{tabular}

Data are expressed as the means $\pm S D$, medians $\left(\mathrm{P}_{25}, \mathrm{P}_{75}\right)$ or percentages.

Central obesity is defined as $\geq 80 \mathrm{~cm}$ for men and $\geq 90 \mathrm{~cm}$ for women; hypertension, diabetes and dyslipidaemia were diagnosed before the study.

DBP, diastolic blood pressure; HDL-C, high-density lipoprotein cholesterol; LDL-C, low-density lipoprotein cholesterol; SBP, systolic blood pressure.

Ten-year probability of developing CHD according to the MetS status

The Framingham risk algorithm was used to estimate the 10-year probability of developing CHD. The distributions of the 10-year estimated risk of developing CHD based on the three different definitions of MetS were compared (table 4). Among those with MetS, based on the CDS criteria, $39.4 \%$ had a 10-year CHD risk of $6 \%$ (low), $6.7 \%$ had a 10 -year CHD risk of $6 \%-10 \%$ (moderate), $7.2 \%$ had a 10-year CHD risk of $10 \%-20 \%$ (moderately high) and $7.2 \%$ had a 10 -year CHD risk of $20 \%$ (high). The remaining $39.4 \%$ of participants with MetS had diabetes and/or CVD. In contrast, among those without MetS, a considerably higher proportion had a low risk $(85.0 \%)$, and lower proportions had a moderate (3.3\%), moderately high $(5.5 \%)$, or high risk $(1.6 \%)$ or had diabetes and/or CVD $(4.7 \%)\left(\chi^{2}=157.65, p<0.001\right)$. Similar heterogeneity in those with MetS and those without MetS was found based on the revised NCEP-ATP III criteria $\left(\chi^{2}=45.17\right.$, $\mathrm{p}<0.001)$ and the IDF criteria $\left(\chi^{2}=306.15, \mathrm{p}<0.001\right)$. Of those with MetS, based on the revised NCEP-ATP III criteria and the IDF criteria, $67.5 \%$ and $74.2 \%$ had a low risk, $3.5 \%$ and $3.0 \%$ had a moderate risk, $5.2 \%$ and $3.3 \%$ had a moderately high risk and $23.8 \%$ and $19.5 \%$ had a high risk or had diabetes and/or CVD, respectively. There were no significant differences in the CHD risk distributions of those with MetS based on the revised NCEP-ATP III criteria and the IDF criteria $\left(\chi^{2}=5.36, p=0.252\right)$, while a significant difference was observed based on the CDS criteria (with the revised NCEP-ATP III criteria: $\chi^{2}=45.71$, with IDF: $\chi^{2}=62.69$, all $p<0.001$, figure 2 ).

We further compared the distribution of the 10-year estimated risk of developing CHD based on the three different MetS definitions in the men and women (figure 3). There were no significant differences in the distribution of the 10-year estimated risk of developing CHD in men with MetS among the three definitions, except for between the revised NCEP-ATP III criteria and the CDS criteria $\left(\chi^{2}=17.41, \mathrm{p}=0.002\right)$. As shown in figure 3 , a significant difference was found in the 10-year risk in women with MetS based on the CDS definition and the remaining definitions (with revised NCEP-ATP III criteria: $\chi^{2}=25.33$, with IDF: $\chi^{2}=37.09$, all $p<0.001$ ), while no significant difference was found based on the revised NCEP-ATP III criteria and the IDF criteria $\left(\chi^{2}=37.09\right.$, $\mathrm{p}=0.245$ ). Compared with the women, a higher CHD risk was found in the men using all three definitions (revised NCEP-ATP III: $\chi^{2}=72.83$; IDF $: \chi^{2}=63.60 ;$ CDS: $\chi^{2}=23.84$; all $\mathrm{p}<0.001$, figure 3 ).

\section{DISCUSSION}

This study shows that the prevalence of MetS and the distribution of the 10-year estimated risk of developing CHD vary depending on how MetS is defined. In this study, the difference among the revised NCEP-ATP III, IDF and CDS criteria was evaluated. The 10-year risk of developing CHD was significantly higher in the participants 


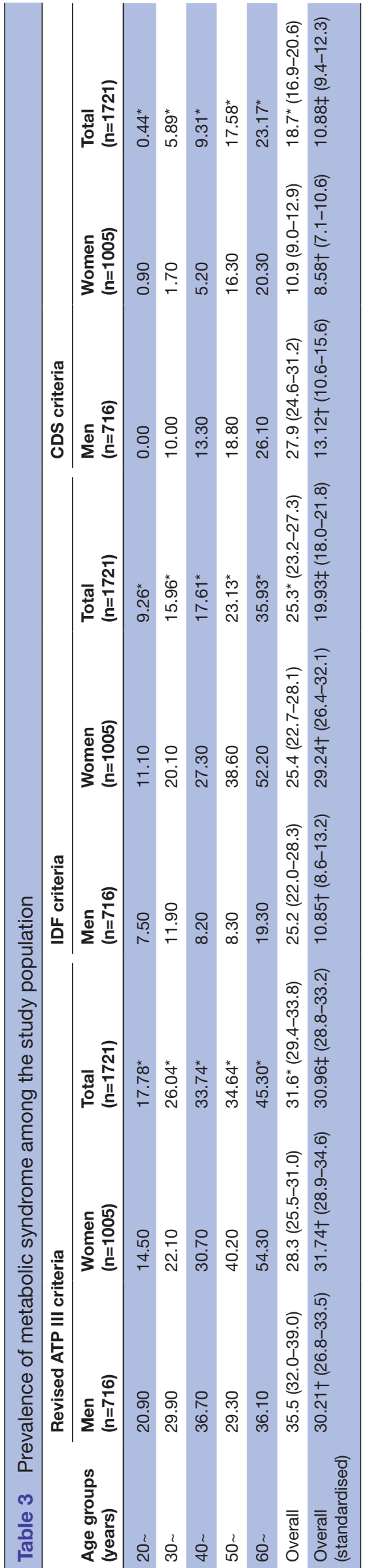

\section{Revised ATP III criteria}

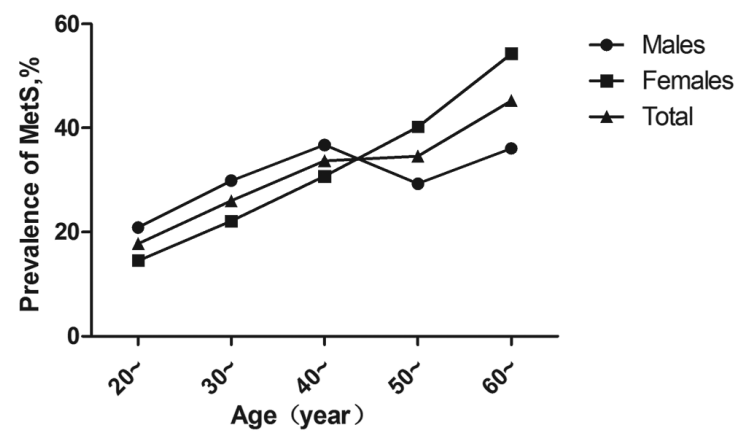

IDF criteria

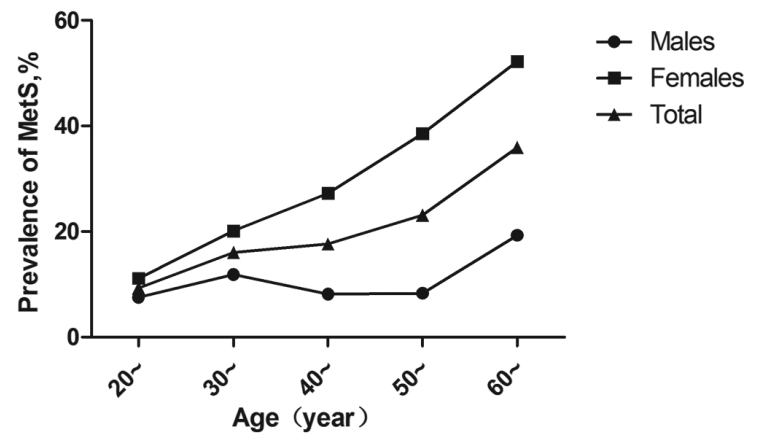

CDS criteria

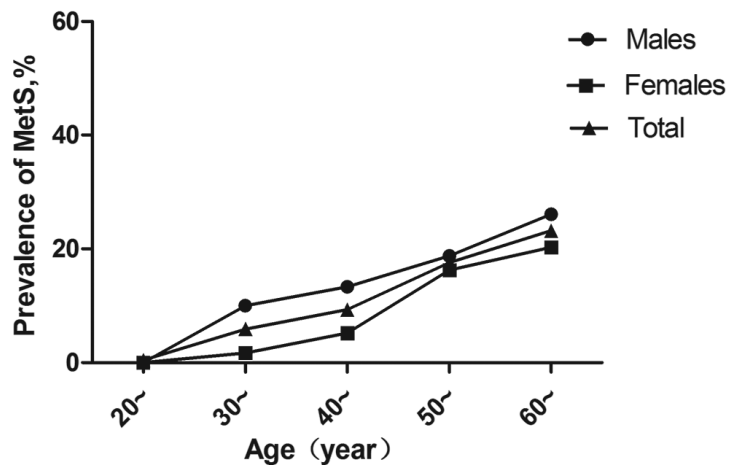

Figure 1 Prevalence of metabolic syndrome (MetS) among adults aged 20 to 80 years in this study area. ATP, Adult Treatment Panel; CDS, Chinese Diabetes Society; IDF, International Diabetes Federation.

with MetS than that in the participants without MetS, and all three definitions demonstrated better performance in reflecting the risk of developing CHD in men than in women. Compared with the other criteria, the participants with MetS based on the CDS criteria had a higher 10-year CHD risk; however, the CDS criteria also led to the lowest prevalence of MetS.

The previous study have examined the ability of different MetS definitions in predicting CVDs. ${ }^{13-17}$ However, to the best of our knowledge, the findings were inconsistent. Similarly, this study was not the first to estimate the 10-year probability in individuals with MetS based on the Framingham risk algorithm. ${ }^{12}{ }^{18}$ Suzuki et al ${ }^{18}$ used the Framingham risk score rather than the 10-year probability of developing CHD to compare the differences among four different MetS definitions. Their results showed that the risk score in men with MetS was significantly higher by 
Table 4 Distribution of the 10-year estimated risk of developing CHD based on the three definitions of MetS

\begin{tabular}{|lccc}
\hline & $\begin{array}{l}\text { Revised } \\
\text { ATP III } \\
\text { criteria }\end{array}$ & $\begin{array}{l}\text { IDF } \\
\text { criteria }\end{array}$ & $\begin{array}{l}\text { CDS } \\
\text { criteria }\end{array}$ \\
\hline MetS (+) & & & \\
\hline Low (<6\%) & 67.5 & 74.2 & 39.4 \\
\hline Moderate (6\%-10\%) & 3.5 & 3 & 6.7 \\
\hline Moderate high (10\%-20\%) & 5.2 & 3.3 & 7.2 \\
\hline High (>20\%) & 3.7 & 3.5 & 7.2 \\
\hline DM/CVD & 20.1 & 16 & 39.4 \\
\hline MetS (-) & & & \\
\hline Low (<6\%) & 86.1 & 81.9 & 85 \\
\hline Moderate (6\%-10\%) & 3.7 & 3.8 & 3.3 \\
\hline Moderate high (10\%-20\%) & 5.9 & 6.3 & 5.5 \\
\hline High (>20\%) & 1.4 & 1.8 & 1.6 \\
\hline DM/CVD & 2.9 & 6.2 & 4.7 \\
\hline P values & $<0.001$ & $<0.001$ & $<0.001$ \\
\hline
\end{tabular}

$P$ value, based on a comparison of the distributions of risk groups between those with and those without metabolic syndrome. ATP, Adult Treatment Panel; CDS, Chinese Diabetes Society; CVD, cardiovascular disease; DM, diabetes mellitus; IDF, International Diabetes Federation; MetS, metabolic syndrome.

threefold than that in women with MetS based on all four diagnostic criteria. However, the results failed to accurately compare the difference between men and women because women are required to have a higher score in each risk category. Therefore, in the present study, we compared the distribution of the 10-year estimated risk of developing CHD between men and women. Our study revealed that all three evaluated definitions of MetS had better performance in reflecting the 10-year CHD risk in men than in women. Furthermore, similar to studies conducted in other populations, ${ }^{19}{ }^{20}$ there were significant differences in the prevalence of MetS between the men and women. A greater number of women met the diagnostic criteria of MetS using the IDF criteria, while the CDS criteria led to a greater number of men having MetS. There was no significant difference in the prevalence of MetS between the women and men based on the revised NCEP-ATP III criteria. The finding that the 10-year probability of developing CHD in men differed based on the definition of MetS is consistent with the findings of previous studies. Mak $e t a l^{21}$ suggested that the adverse impact of MetS was greater among men than women, which is consistent with another study. ${ }^{15}$ Therefore, the impacts of various risk factors on CVDs and their outcomes appear to differ according to sex in patients with MetS. ${ }^{22}$ Notably, different forms of obesity have different impacts on CVD risk. In particular, android obesity, which is more common in men and postmenopausal women, ${ }^{23}$ is associated with future cardiovascular events. ${ }^{24}$ This sex difference may also be due to other characteristics of the subjects, such as age and smoking status. In contrast, some studies ${ }^{51325}$ have suggested that all definitions of MetS (NCEP-ATP III, IDF, AHA/NHLBL and JIS) are more predictive of the CHD risk in women than in men.

Among the definitions of MetS evaluated in the current study, the IDF criteria identified fewer participants $(19.93 \%)$ as having MetS than the revised NCEP-ATP III criteria $(30.96 \%)$, but this lower prevalence did not translate into better performance. There was no significant difference in the distribution of the 10-year risk of developing CHD between the revised NCEP-ATP III and the IDF criteria. This finding is consistent with the results of previous studies in which similar risks of CVDs were reported with different levels of sensitivity depending on the definition of MetS. ${ }^{13} 2627$ The lower prevalence based on the IDF criteria may be due to the requirement

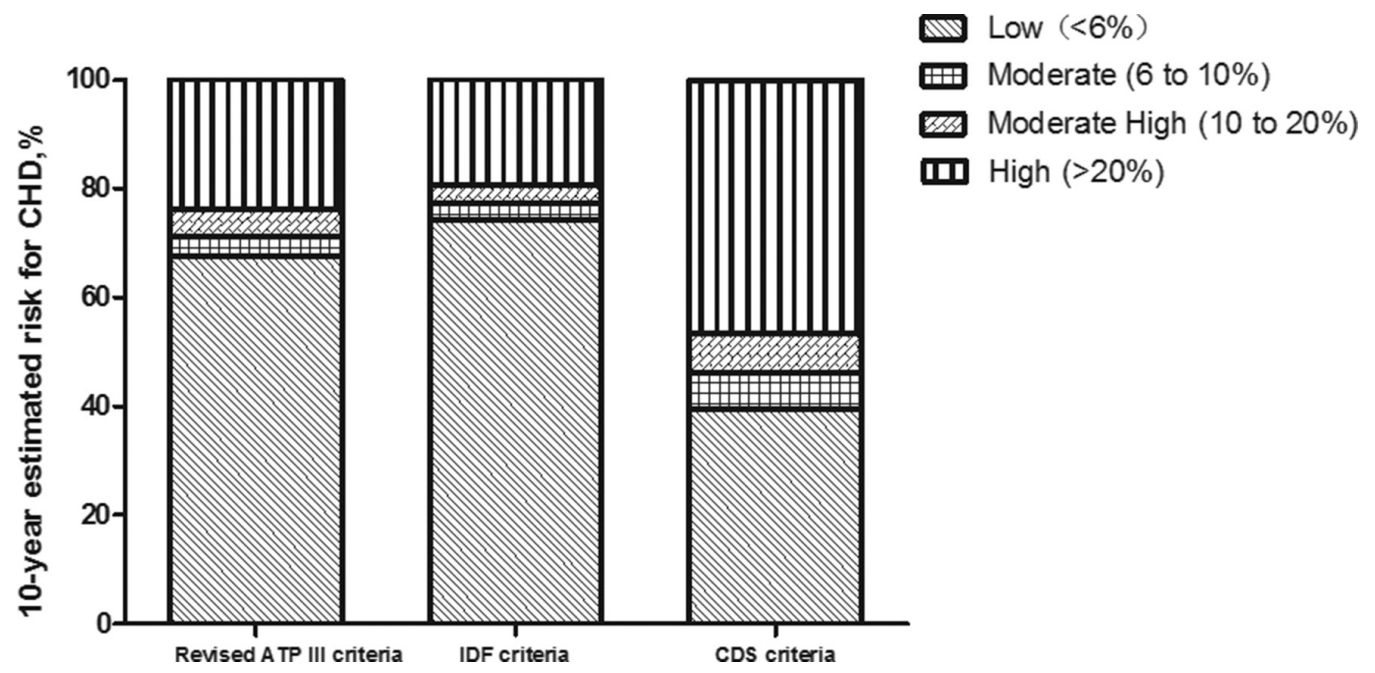

Figure 2 The distribution of the 10-year estimated risk for coronary heart disease (CHD) in individuals with metabolic syndrome (MetS) based on the three different definitions of MetS. The risk categories are as follows: low $(<6 \%)$, moderate $(6 \%$ to $10 \%)$, moderately high (10\% to $20 \%$ ) and high ( $>20 \%$ or history of diabetes or cardiovascular disease). ATP, Adult Treatment Panel; CDS, Chinese Diabetes Society; IDF, International Diabetes Federation. 

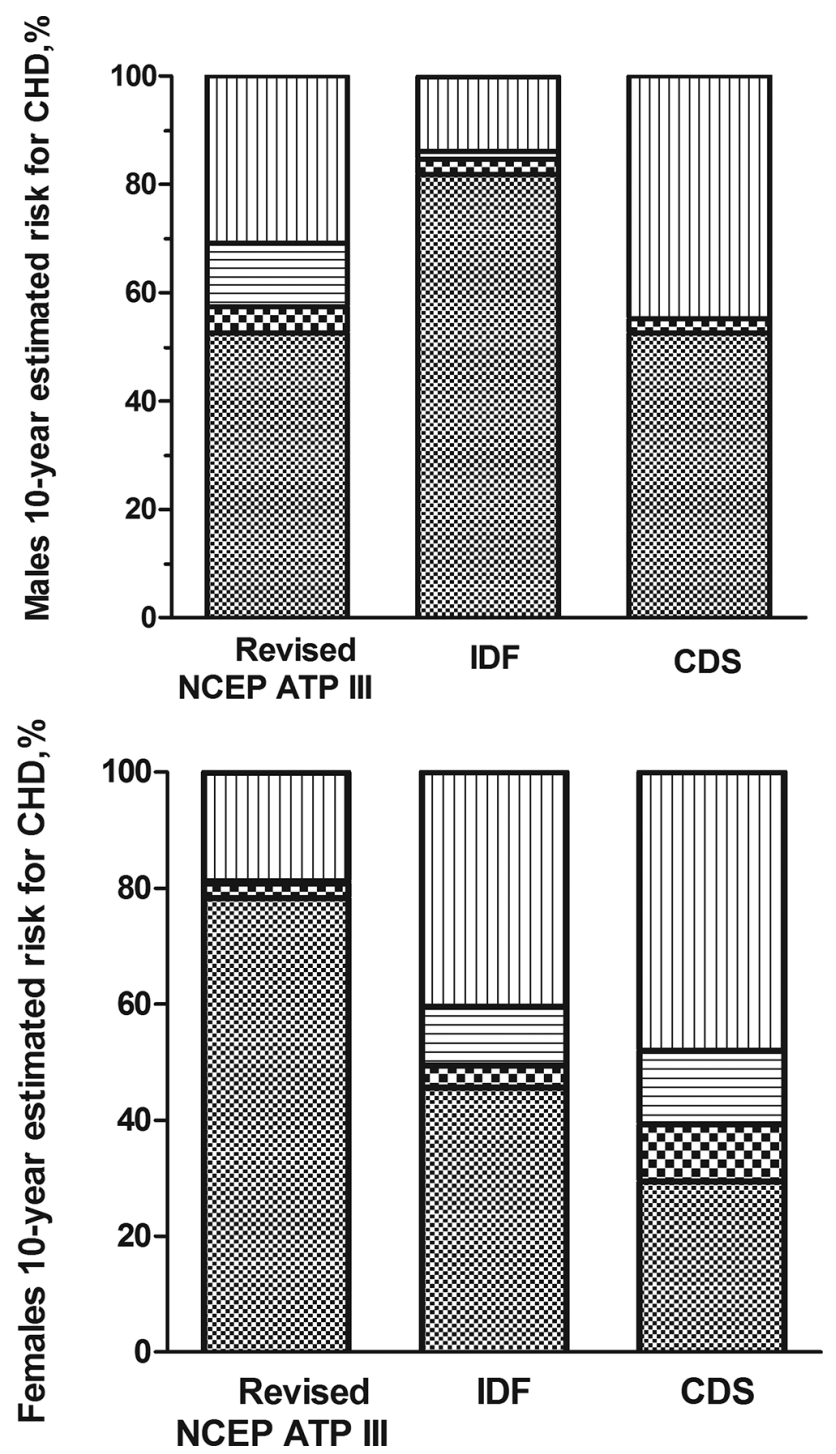

of central obesity for the diagnosis of MetS, even though these criteria share the same components and same cut-off values. Compared with the revised ATP III criteria, this demand decreases the number of individuals satisfying the criteria for MetS under the IDF criteria. In addition, if the threshold value of abdominal obesity differs among different MetS definitions, the discrepancy in prevalence may be reversed. For instance, Scuteri et al ${ }^{19}$ reported that the prevalence of MetS based on the IDF criteria was higher than that based on the ATP criteria, which may result from the lower waist circumstance threshold values applied to the European population by the IDF. Notably, a recent cohort study conducted by Keihani ${ }^{28}$ showed that abdominal obesity and the presence of metabolic derangements are both relevant risk factors for future CVD. Similar results were found in another study by Zhao et $a l,{ }^{29}$ who compared the long-term risk of CVDs between patients with MetS with or without central obesity. These authors found that most patients with MetS (78\%) had central obesity, and no significant difference was observed in the 10-year absolute and relative risk of CHD and ischaemic CVD events between the two MetS groups. This finding highlights the fact that focusing on abdominal obesity while ignoring the other components of MetS may not be ideal. Another study ${ }^{16}$ using an receiver operating characteristic curve and Cox regression analyses showed that the ATP III criteria better predicted CVD than the IDF criteria.

Compared with the other criteria, the CDS criteria led to the lowest prevalence of MetS and the highest 10-year probability of developing CHD in the current study. Our findings are partially consistent with the results of previous studies in which CDS had the highest specificity in identifying MetS in a Chinese population based on a 
6.3-year cohort study. ${ }^{16}$ However, despite the high specificity, the study also found that the CDS criteria had the lowest sensitivity among the three definitions, and more than $50 \%$ of patients may be misdiagnosed. More subjects were diagnosed with obesity (BMI $\geq 25 \mathrm{~kg} / \mathrm{m}^{2}$ ) than central obesity ( $32.31 \%$ vs $24.17 \%$ ). Therefore, the lowest prevalence and the highest risk of developing CHD are mainly caused by the thresholds of high blood pressure and elevated blood glucose in the CDS criteria, which are higher than those in the other criteria. However, discussing the superiority of the MetS definition that adopts BMI or WC as an index of adiposity is necessary. Some studies posit that WC is a more advantageous index of adiposity. According to Scuteri et $a l^{30} \mathrm{WC}$ is a significant predictor of new-onset MetS. In addition, Scuteri et $a l^{31}$ indicated that WC correlated with arterial properties better than BMI and that as the WC increased, the arterial structure and function significantly changed within each BMI quartile, even though the cluster of MetS including abdominal adiposity has been consistently associated with arterial damage. $^{32} 33$

The strength of our study should be mentioned. The complex, multistage probability sample design is fairly representative of the Chinese population in Shenzhen. In addition, the percentage of missing data is generally low. However, there are several limitations to our study. First, although the original Framingham CHD risk assessment has been validated in previous studies, ${ }^{34}$ the algorithm does not include obesity and cardiorespiratory fitness, ${ }^{35-37}$ which could have potentially influenced the risk estimation. Furthermore, a previous report found that the Framingham algorithm overestimates the risk of CHD in the Chinese population. ${ }^{38}$ Second, our analysis was based on cross-sectional data; therefore, we were unable to calculate positive and negative predictive values for CHD or determine which MetS definition is the most predictive of the development of CHD. Thus, the results should be interpreted with caution. Further studies conducted in China, especially longitudinal studies, are needed to determine which MetS definition is best suited for predicting CHD.

This study contributes to the body of evidence showing that differences exist in the prevalence and distribution of the 10-year estimated risk of developing CHD depending on the definition of MetS. Among the definitions evaluated (the revised NCEP-ATP III, IDF and CDS criteria), the CDS criteria led to the highest 10 -year probability of developing CHD and the lowest prevalence of MetS. A significant finding of this study was that all three definitions of MetS had better performance in men compared with women.

\footnotetext{
Author affiliations

${ }^{1}$ Department of Nutrition and Food Hygiene, School of Public Health, Tongji Medical College, Huazhong University of Science and Technology, Wuhan, China

${ }^{2}$ Hubei Key Laboratory of Food Nutrition and Safety, Huazhong University of Science and Technology Tongji Medical College, Wuhan, China

${ }^{3}$ Clinical laboratory, Shenzhen Centre for Chronic Disease Control, Shenzhen, China
}

${ }^{4}$ Department of chronic noncommunicable diseases, Nanshan Centre for Chronic Disease Control, Shenzhen, China

Acknowledgements The authors are grateful to the participants who participated in this survey and to the staff of the Community Health Service Center.

Contributors The author contributions were as follows: XP and LH conceived and designed the study and critically revised the manuscript; JZ analysed the data and wrote the paper; QG and JW participated in the laboratory assay; and MZ, JM, $\mathrm{CW}$ and $\mathrm{HC}$ collected the data and revised the manuscript. All authors read and approved the final version of the manuscript.

Funding The present study was jointly supported by the National Natural Science Foundation of China (no. 81573149), Projects Funded of Health and Family Commission of Shenzhen Municipality (no. 201502017 and no. SZSJ2017001) and Project Funded of Nanshan District, Shenzhen Science and Technology Innovation Bureau (no. 2015064).

Competing interests None declared.

Patient consent Obtained.

Ethics approval The study was approved by the Ethics Committee of the Shenzhen Nanshan Center for Chronic Disease Control.

Provenance and peer review Not commissioned; externally peer reviewed. Data sharing statement № additional data are available.

Open access This is an open access article distributed in accordance with the Creative Commons Attribution Non Commercial (CC BY-NC 4.0) license, which permits others to distribute, remix, adapt, build upon this work non-commercially, and license their derivative works on different terms, provided the original work is properly cited, appropriate credit is given, any changes made indicated, and the use is non-commercial. See: http://creativecommons.org/licenses/by-nc/4.0/.

\section{REFERENCES}

1. Sattar N, Gaw A, Scherbakova O, et al. Metabolic syndrome with and without C-reactive protein as a predictor of coronary heart disease and diabetes in the West of Scotland Coronary Prevention Study. Circulation 2003;108:414-9.

2. Ford ES. Risks for all-cause mortality, cardiovascular disease, and diabetes associated with the metabolic syndrome: a summary of the evidence. Diabetes Care 2005;28:1769-78.

3. Noda H, Iso H, Saito I, et al. The impact of the metabolic syndrome and its components on the incidence of ischemic heart disease and stroke: the Japan public health center-based study. Hypertens Res 2009;32:289-98.

4. Mottillo S, Filion KB, Genest J, et al. The metabolic syndrome and cardiovascular risk a systematic review and meta-analysis. J Am Coll Cardiol 2010;56:1113-32.

5. Gami AS, Witt BJ, Howard DE, et al. Metabolic syndrome and risk of incident cardiovascular events and death: a systematic review and meta-analysis of longitudinal studies. J Am Coll Cardiol 2007;49:403-14.

6. Esteghamati A, Hafezi-Nejad N, Sheikhbahaei S, et al. Risk of coronary heart disease associated with metabolic syndrome and its individual components in Iranian subjects: a matched cohort study. $J$ Clin Lipidol 2014;8:279-86.

7. National Cholesterol Education Program (NCEP) Expert Panel on Detection, Evaluation, and Treatment of High Blood Cholesterol in Adults (Adult Treatment Panel III). Third Report of the National Cholesterol Education Program (NCEP) Expert Panel on Detection, Evaluation, and Treatment of High Blood Cholesterol in Adults (Adult Treatment Panel III) final report. Circulation 2002;106:3143-421.

8. Alberti KG, Zimmet P, Shaw J, et al. The metabolic syndrome-a new worldwide definition. Lancet 2005;366:1059-62.

9. Anon. Metabolic syndrome study cooperation group of Chinese diabetes society. Chin J Diabetes Mellitus 2004;12:156-61.

10. Alberti KG, Eckel RH, Grundy SM, et al. Harmonizing the metabolic syndrome: a joint interim statement of the International Diabetes Federation Task Force on Epidemiology and Prevention; National Heart, Lung, and Blood Institute; American Heart Association; World Heart Federation; International Atherosclerosis Society; and International Association for the Study of Obesity. Circulation 2009;120:1640-5.

11. Grundy SM, Cleeman JI, Daniels SR, et al. Diagnosis and management of the metabolic syndrome: an American 
Heart Association/National Heart, Lung, and Blood Institute scientific statement: Executive Summary. Crit Pathw Cardiol 2005;4:198-203

12. Hoang KC, Ghandehari H, Lopez VA, et al. Global coronary heart disease risk assessment of individuals with the metabolic syndrome in the U.S. Diabetes Care 2008;31:1405-9.

13. Hosseinpanah F, Asghari G, Barzin M, et al. Prognostic impact of different definitions of metabolic syndrome in predicting cardiovascular events in a cohort of non-diabetic Tehranian adults. Int J Cardiol 2013;168:369-74.

14. Kastorini CM, Panagiotakos DB, Georgousopoulou EN, et al. Metabolic syndrome and 10-year cardiovascular disease incidence: The ATTICA study. Nutr Metab Cardiovasc Dis 2016;26:223-31.

15. Qiao Q. DECODE Study Group. Comparison of different definitions of the metabolic syndrome in relation to cardiovascular mortality in European men and women. Diabetologia 2006;49:2837-46.

16. Zhou H, Guo ZR, Yu LG, et al. Evidence on the applicability of the ATPIII, IDF and CDS metabolic syndrome diagnostic criteria to identify CVD and T2DM in the Chinese population from a 6.3year cohort study in mid-eastern China. Diabetes Res Clin Pract 2010;90:319-25.

17. Scuteri A, Najjar SS, Morrell CH, et al. The metabolic syndrome in older individuals: prevalence and prediction of cardiovascular events: the Cardiovascular Health Study. Diabetes Care 2005;28:882-7.

18. Suzuki T, Zeng Z, Zhao B, et al. Comparison of coronary heart disease risk among four diagnostic definitions of metabolic syndrome. J Endocrinol Invest 2016;39:1337-46.

19. Scuteri A, Najjar SS, Orru' M, et al. Age- and gender-specific awareness, treatment, and control of cardiovascular risk factors and subclinical vascular lesions in a founder population: the SardiNIA Study. Nutr Metab Cardiovasc Dis 2009;19:532-41.

20. Scuteri A, Laurent S, Cucca F, et al. Metabolic syndrome across Europe: different clusters of risk factors. Eur J Prev Cardiol 2015;22:486-91.

21. Mak KH, Ma S, Heng D, et al. Impact of sex, metabolic syndrome, and diabetes mellitus on cardiovascular events. Am J Cardiol 2007;100:227-33.

22. Regitz-Zagrosek V, Lehmkuhl E, Weickert MO. Gender differences in the metabolic syndrome and their role for cardiovascular disease. Clin Res Cardiol 2006;95:136-47.

23. Williams CM. Lipid metabolism in women. Proc Nutr Soc 2004;63:153-60.

24. Ibrahim MM. Subcutaneous and visceral adipose tissue: structural and functional differences. Obes Rev 2010:11:11-18.
25. Wang $\mathrm{C}, \mathrm{Hou} \mathrm{X}, \mathrm{Bao} \mathrm{Y}$, et al. The metabolic syndrome increased risk of cardiovascular events in Chinese-a community based study. Int $J$ Cardiol 2010;139:159-65.

26. Mancia G, Bombelli M, Facchetti R, et al. Impact of different definitions of the metabolic syndrome on the prevalence of organ damage, cardiometabolic risk and cardiovascular events. $J$ Hypertens 2010;28:999-1006.

27. Nilsson PM, Engström G, Hedblad B. The metabolic syndrome and incidence of cardiovascular disease in non-diabetic subjects-a population-based study comparing three different definitions. Diabet Med 2007;24:464-72.

28. Keihani S, Hosseinpanah F, Barzin M, et al. Abdominal obesity phenotypes and risk of cardiovascular disease in a decade of follow-up: the Tehran Lipid and Glucose Study. Atherosclerosis 2015;238:256-63.

29. Zhao D, Grundy SM, Wang W, et al. Ten-year cardiovascular disease risk of metabolic syndrome without central obesity in middle-aged chinese. Am J Cardiol 2007;100:835-9.

30. Scuteri A, Morrell CH, Najjar SS, et al. Longitudinal paths to the metabolic syndrome: can the incidence of the metabolic syndrome be predicted? The Baltimore Longitudinal Study of Aging. J Gerontol A Biol Sci Med Sci 2009;64:590-8.

31. Scuteri A, Orru' M, Morrell $\mathrm{CH}$, et al. Associations of large artery structure and function with adiposity: effects of age, gender, and hypertension. The SardiNIA Study. Atherosclerosis 2012;221:189-97.

32. Scuteri A, Cunha PG, Rosei EA, et al. Arterial stiffness and influences of the metabolic syndrome: a cross-countries study. Atherosclerosis 2014;233:654-60.

33. Scuteri A, Naijar SS, Orru' M, et al. The central arterial burden of the metabolic syndrome is similar in men and women: the SardiNIA Study. Eur Heart J 2010;31:602-13.

34. D'Agostino RB, Grundy S, Sullivan LM, et al. Validation of the Framingham coronary heart disease prediction scores: results of a multiple ethnic groups investigation. JAMA 2001;286:180-7.

35. Lavie CJ, Deedwania P, Ortega FB. Obesity is rarely healthy. Lancet Diabetes Endocrinol 2018;6:678-9.

36. Kennedy AB, Lavie CJ, Blair SN. Fitness or Fatness: Which Is More Important? JAMA 2018;319:231-2.

37. Deedwania P, Lavie CJ, Dangers LCJ. Dangers and Long-Term Outcomes in Metabolically Healthy Obesity: The Impact of the Missing Fitness Component. J Am Coll Cardiol 2018;71:1866-8.

38. Liu J, Hong Y, D'Agostino RB, et al. Predictive value for the Chinese population of the Framingham CHD risk assessment too compared with the Chinese Multi-Provincial Cohort Study. JAMA 2004;291:2591-9. 